\title{
Technè
}

La science au service de l'histoire de l'art et de la préservation des biens culturels

$40 \mid 2014$

Thérapéia. Polychromie et restauration de la sculpture dans l'Antiquité

\section{Restauration et entretien de la statuaire dans les temples égyptiens : un aperçu}

Restoration and upkeep of statues in Egyptian temples: an overview

Marie-Paule Jung

\section{(2) OpenEdition}

Journals

Édition électronique

URL : https://journals.openedition.org/techne/3402

DOI : 10.4000/techne.3402

ISSN : 2534-5168

Éditeur

C2RMF

Édition imprimée

Date de publication : 26 novembre 2014

Pagination : 46-52

ISBN : 978-2-7118-6218-4

ISSN : 1254-7867

Référence électronique

Marie-Paule Jung, «Restauration et entretien de la statuaire dans les temples égyptiens : un aperçu », Technè [En ligne], 40 | 2014, mis en ligne le 24 juillet 2020, consulté le 09 février 2022. URL : http:// journals.openedition.org/techne/3402 ; DOI : https://doi.org/10.4000/techne.3402

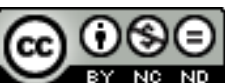

La revue Technè. La science au service de l'histoire de l'art et de la préservation des biens culturels est mise à disposition selon les termes de la Licence Creative Commons Attribution - Pas d'Utilisation

Commerciale - Pas de Modification 4.0 International. 


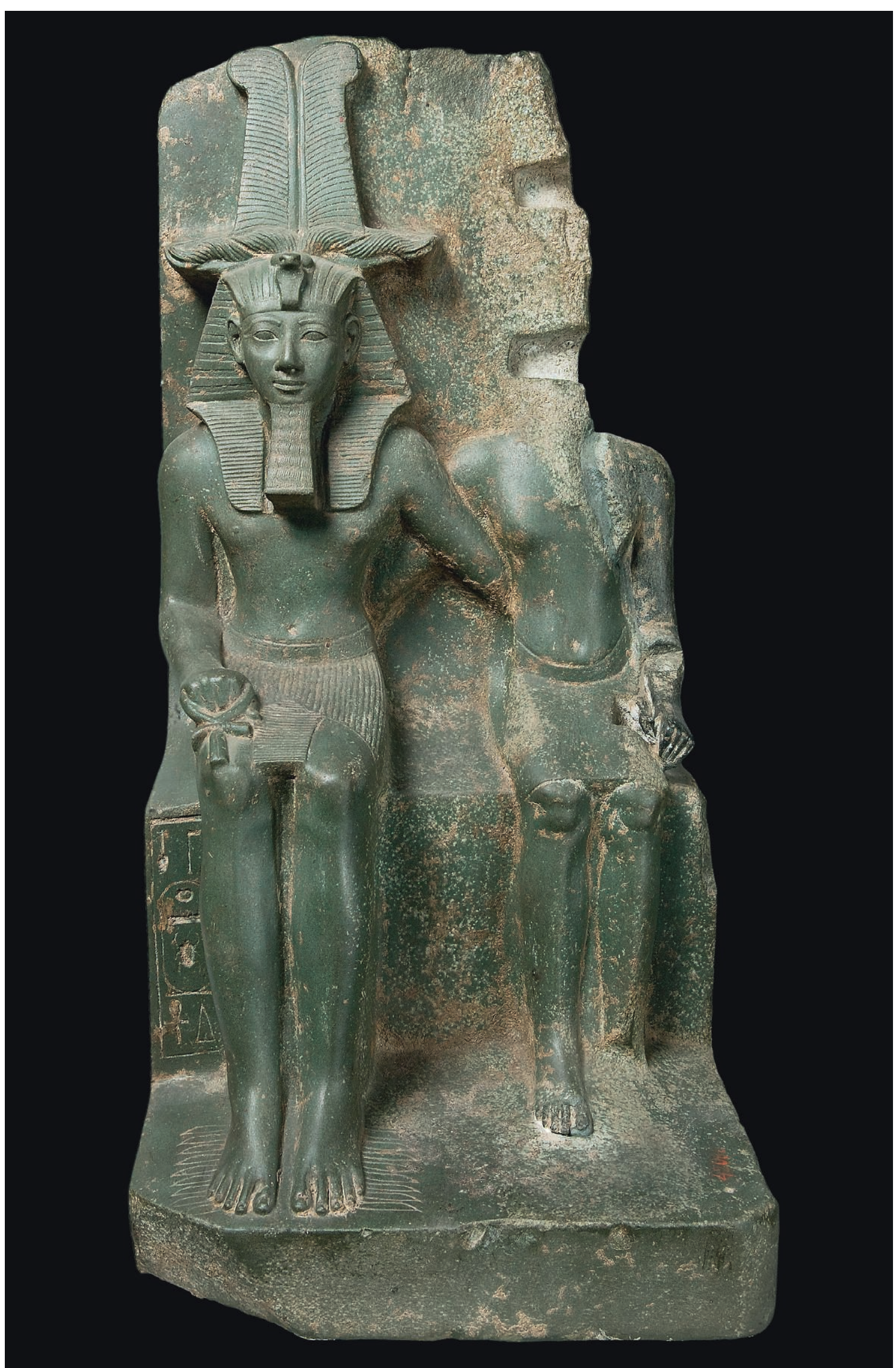

Fig. 1. Groupe de Thoutmosis III et Amon découvert dans la «Cachette» de Karnak, H. 68 cm ; 1. 31,5 cm. (C) IFAO, Ibrahim Mohammed (Ihab). 
Marie-Paule Jung

\title{
Restauration et entretien de la statuaire dans les temples égyptiens : un aperçu
}

\author{
Restoration and upkeep of statues in Egyptian \\ temples: an overview
}

Résumé. À travers la présentation de quelques exemples, cet article propose un aperç des techniques de restauration, réparation et entretien des statues placées dans les temples égyptiens ; les valeurs pouvant être attribuées à ces interventions dans certains contextes sont également abordées.

Mots-clés. Égypte pharaonique, histoire de la restauration, statuaire, mobilier de temple, Karnak.

\section{Introduction}

Les statues égyptiennes ont connu des destins variés: certaines furent placées dans des tombes, lors de funérailles, et y demeurèrent jusqu'au jour de leur découverte; d'autres, au contraire, ont connu une vie plus mouvementée, en particulier celles exposées dans les temples, où elles sont parfois restées pendant des siècles. Confrontées aux outrages du temps, elles ont pu être déplacées, remployées, usurpées et subir, au cours de leurs différentes phases d'usage, un dommage accidentel ou volontaire, parfois suivi d'une restauration. De nombreuses statues témoignent de ces réfections antiques, certaines ayant été conditionnées par la spécificité des images égyptiennes, lesquelles sont avant tout fonctionnelles et dotées d'une capacité d'action propre en tant que substitut du corps d'un personnage ${ }^{1}$.

Les termes modernes susceptibles d'être utilisés pour décrire ces opérations, tels que «restauration» ou «réparation ${ }^{2}$ » permettent difficilement de retranscrire fidèlement la réalité de ces interventions antiques, commandées par des conceptions qui différent des nôtres en bien des aspects.

Nous proposons d'employer le mot «réparation» et ses synonymes («réfection», «remise en état», «rénovation»), dans le cas d'interventions visant à rétablir, de façon plus ou moins fidèle, un objet dans un état matériel antérieur, et de réserver le terme de «restauration » pour désigner des interventions vouées en priorité à restituer à un objet sa capacité à agir, plutôt que son aspect initial ${ }^{3}$. Cependant, la frontière entre ces deux types d'interventions n'est pas hermétique et souvent difficile à établir.

Le vocabulaire égyptien ne semble pas opérer de distinctions formelles et les égyptologues proposent, pour un même terme, plusieurs traductions différentes: ainsi le terme sm3wy,
Abstract. By presenting a number of examples, this article offers an overview of the techniques used in the restoration, repairing and upkeep of statues placed in Egyptian temples. The values possibly attributed to these operations in certain contexts will also be discussed.

Keywords. Pharaonic Egypt, restoration history, statuary, temple furniture, Karnak.

apparaissant fréquemment dans la littérature égyptienne et s'appliquant à de nombreux objets différents (tels que des édifices, des sculptures, des barques processionnelles, mais aussi le corps, le nom ou les offrandes) est aussi bien rendu par « restaurer» que par « rénover» ou encore «renouveler ${ }^{4}$ ». Les sources textuelles sont donc délicates à interpréter, d'autant plus qu'il s'agit le plus souvent de textes destinés à mettre en avant une intervention commanditée par leur auteur. L'objectivité et les questions techniques y occupent alors peu de place. Par ailleurs, les inscriptions et les témoignages matériels concernant la réfection des sculptures dans la civilisation pharaonique ont été encore peu étudiés, et seule une analyse systématique de ces témoignages permettrait d'élargir nos connaissances sur les pratiques de restauration des anciens Égyptiens ${ }^{5}$.

\section{Identification des réparations antiques}

La statuaire en pierre pharaonique est le plus souvent monolithique. Des pièces rapportées pouvaient cependant être utilisées dans certains cas, afin de fixer des couronnes par exemple. Une production de statues constituées de plusieurs éléments assemblés est également attestée à la fin de la $\mathrm{XVIII}^{\mathrm{e}}$ dynastie $^{6}$. En dehors de ces cas particuliers, tout assemblage inhabituel est susceptible d'être le résultat d'une remise en état. L'observation des objets est le point de départ de la reconnaissance des réparations antiques. Ces dernières sont souvent bien visibles en raison de la fréquente perte des matériaux ou des éléments sculptés employés pour leur mise en œuvre. Elles se trahissent alors par diverses traces laissées sur la surface de l'objet, tels qu'une découpe nette, des tenons ou des mortaises. Des éléments de restauration sont également 


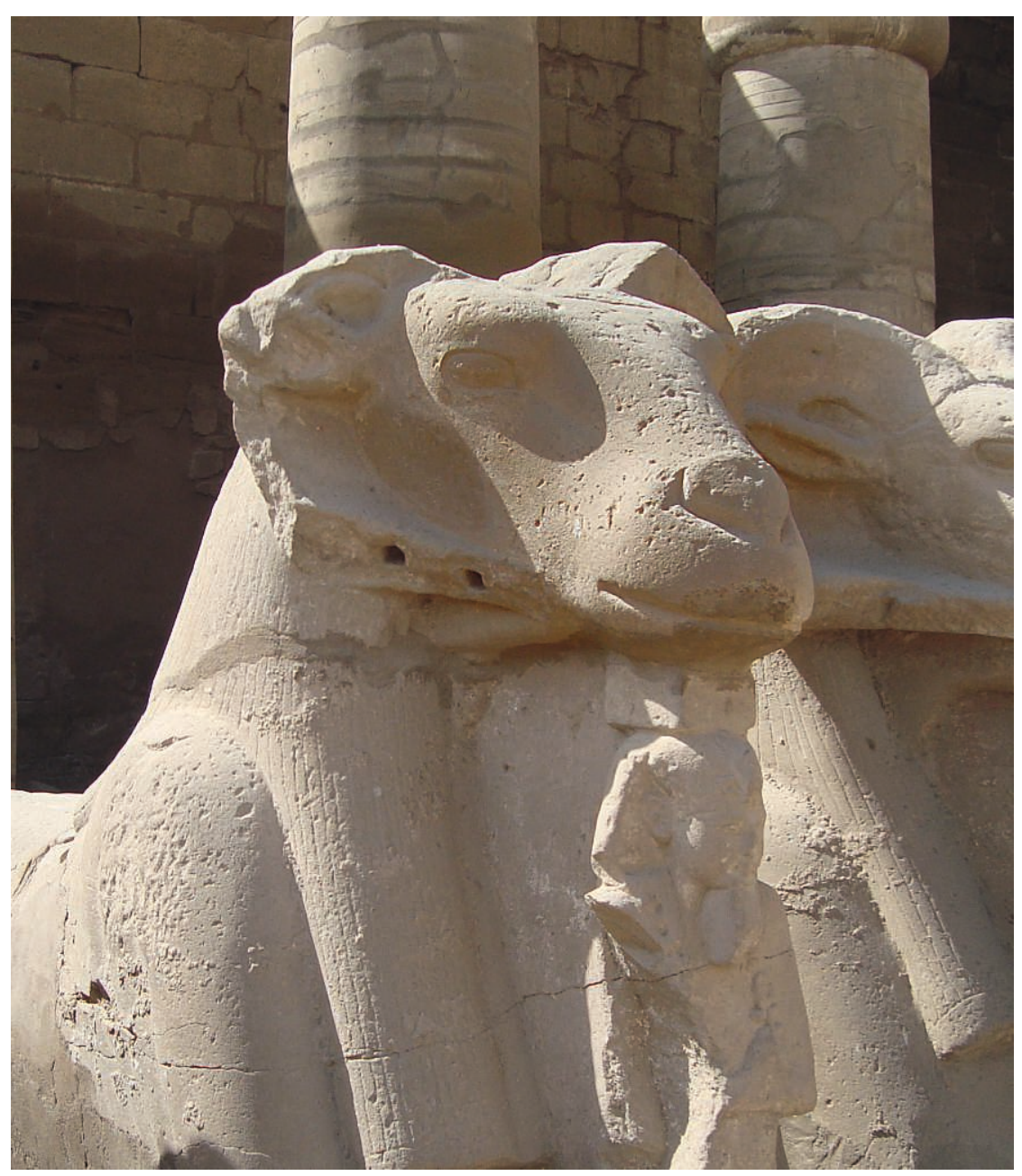

Fig. 2. Détail d'un

criosphinx situé dans la

partie sud de la cour

du I ${ }^{\text {er }}$ pylône du temple

d'Amon de Karnak.

() Marie-Paule Jung.

conservés, isolés ou toujours liés au monument qu'ils complètent (fig. 3) : outre le groupe de Thoutmosis III (fig. 1) présenté ci-dessous, on peut citer une statue d'Amenhotep III, conservée au Metropolitan Museum of Art de New York (22.5.2), comportant deux éléments de restauration fixés avec un système de tenons et mortaises ou encore une statue-cube de Bakenkhonsou du Musée égyptien du Caire (CG 42155) dont le nez a été réparé à l'aide d'un élément rapporté, toujours en place ${ }^{7}$.

Le matériau originel des monuments et ceux employés pour sculpter des pièces de réparation sont le plus souvent de même nature ${ }^{8}$, ou, du moins, d'apparence similaire ${ }^{9}$. Les mortiers employés pour restituer un plus faible volume sont également composés de façon à s'adapter à la couleur de la pierre sur laquelle ils sont placés ${ }^{10}$. Néanmoins, lorsqu'une réfection consiste à appliquer une nouvelle couche de polychromie, la couleur originelle de l'œuvre ne semble pas toujours respectée ${ }^{11}$.

Peu d'éléments permettent de proposer une datation concernant les réfections antiques et il est souvent difficile de distinguer une intervention postérieure à la consécration d'une statue d'une opération résultant d'un accident survenu lors du travail de taille. Certaines statues ont en effet été réparées au cours de leur fabrication. C'est le cas d'une représentation inachevée d'Amenhotep III du musée d'Art égyptien ancien de Louqsor (J. 132). Elle comporte une mortaise en double queue-d'aronde traversant une fissure dans laquelle devait être placé un crampon liant le tout.

Des inscriptions associées aux représentations ou des traces matérielles visibles sur l'œuvre, telles que celles laissées par des dommages caractéristiques d'une agression volontaire, peuvent néanmoins permettre de situer certaines interventions ${ }^{12}$.

Les méthodes de réfection, tout comme les procédés de la taille de pierre, sont d'une grande constance tout au long de la période pharaonique et ne permettent pas de déceler une évolution chronologique des techniques. Les artisans effectuant les rénovations étaient probablement les mêmes que ceux qui créaient les statues, ces deux activités requérant des compétences similaires. Nous disposons de peu d'informations sur ces praticiens, les textes antiques faisant allusion à la réfection de monuments émanant plus volontiers des commanditaires que du personnel chargé de leur exécution. Ces textes relatifs à la réparation de sculptures sont plutôt rares, au contraire des mentions de restaurations architecturales dont les exemples ne manquent pas ${ }^{13}$. 


\section{Rénovation et prévention}

Tout comme les édifices et leur décor gravé ou peint, les sculptures placées dans les temples pouvaient bénéficier de rénovations, isolées ou groupées. Parmi les criosphinx ${ }^{14} \mathrm{de}$ grès placés aujourd'hui de part et d'autre du I ${ }^{\text {er }}$ pylône du temple d'Amon à Karnak, un certain nombre porte des traces de réfection, au niveau des cornes ou de la tête, consistant le plus souvent en un ensemble de petites mortaises circulaires ou quadrangulaires (fig. 2) dans lesquelles étaient probablement placés des faux-tenons en bois permettant la fixation de pièces de réparation; un fragment d'un de ces éléments sculptés reconstituant une partie de la corne gauche de la tête du bélier est encore en place sur l'une des statues (fig. 3). Des criosphinx présentent également des traces de plâtre utilisé pour boucher des dommages de surface, notamment des cupules creusées dans l'Antiquité ${ }^{15}$. Sur certaines de ces sculptures, on a constaté la présence de plusieurs couches de peinture de couleurs différentes appliquées successivement ${ }^{16}$. Tous ces éléments semblent témoigner de plusieurs campagnes de rénovation ${ }^{17}$.

La question des reprises de polychromie sur les monuments égyptiens est délicate à aborder: très peu de témoignages sont actuellement connus. Il est cependant probable que les sculptures, souvent exposées dans les cours de temples sur de très longues durées, bénéficiaient parfois d'un «rafrâ̂chissement» de leurs couleurs, à l'instar de ces criosphinx. Une meilleure connaissance de l'histoire matérielle des objets permet parfois d'identifier des renouvellements de la polychromie, comme dans le cas de la «tête Salt». L'étude de cette sculpture a permis de reconstituer son histoire mouvementée: d'abord conçue en tant qu'élément d'encastrement destiné à compléter une statue acéphale, la tête a par la suite séjourné dans un milieu humide, comme l'atteste un réseau de concrétions visibles sur la surface de la pierre, puis elle a connu une utilisation différente, à l'occasion de laquelle une nouvelle couche de peinture a été appliquée ${ }^{18}$.

Le colosse de granite de Ramsès II, qui se dresse au nord ouest du I ${ }^{\text {er }}$ pylône du temple de Louqsor (fig. 4), illustre quant à lui une mesure de prévention visant à consolider une fissure traversant le côté droit du siège et l'appui dorsal de la sculpture. En effet, des mortaises en queue-d'aronde ont été taillées de part et d'autre du dommage. Elles devaient accueillir des crampons ${ }^{19}$ solidarisant le tout, probablement façonnés en bois recouvert de mortier, afin d'empêcher une éventuelle aggravation du dommage. La sculpture a par ailleurs été réparée en différents emplacements, avec un système de tenons et mortaises, notamment au niveau du coude droit et de l'épaule gauche.

\section{Restaurations d'images divines}

La restauration des représentations divines est particulièrement bien documentée pour l'époque post-amarnienne. Cette période fait suite au règne d'Akhenaton qui instaura un ensemble de réformes politiques et religieuses bouleversant l'équilibre habituel de l'Égypte et se traduisant notamment par la destruction systématique de représentations de divinités - en particulier Amon - au profit du seul culte du dieu Aton. Cet épisode de courte durée engendra de nombreuses destructions que les rois suivants s'efforcèrent d'effacer au moyen d'un vaste programme de restauration, décrit dans la célèbre «stèle de la Restauration » de Toutânkhamon ${ }^{20}$ et illustré par de nombreuses restaurations de reliefs et de statues.

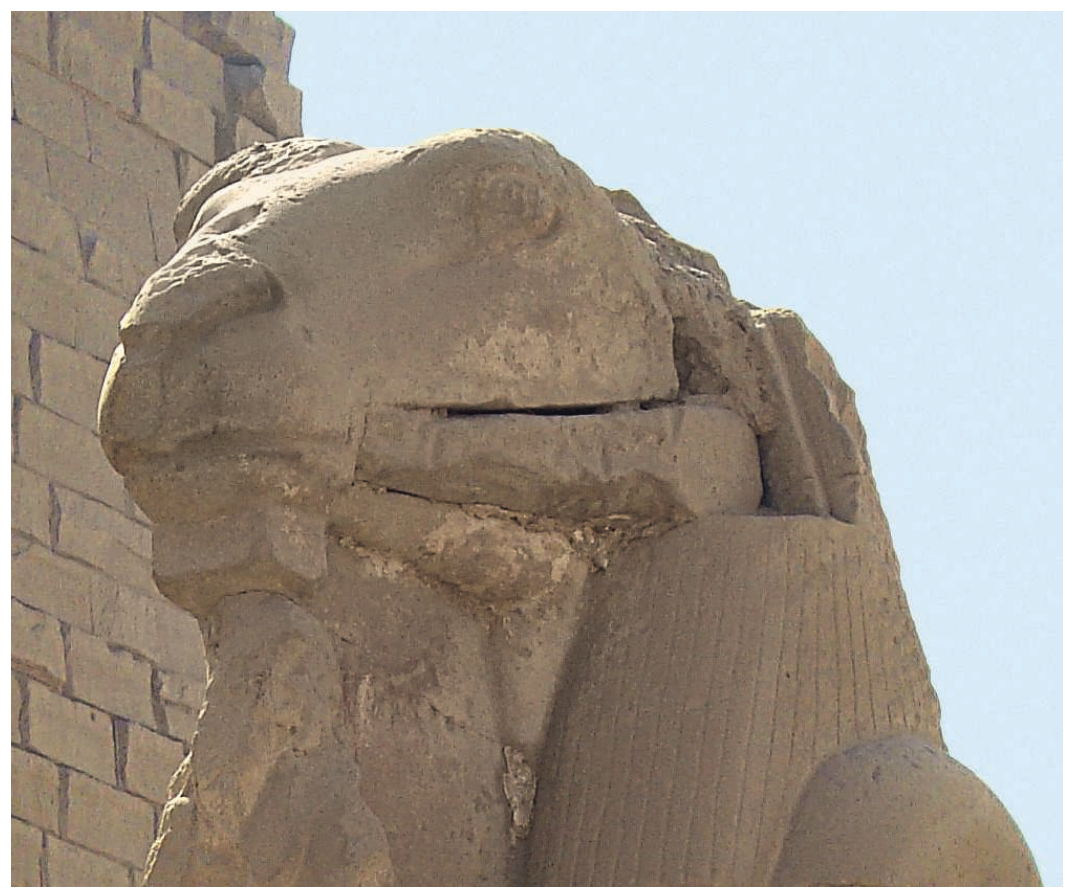

Fig. 3. Détail d'un criosphinx situé au sud-ouest du I ${ }^{\mathrm{er}}$ pylône du temple d'Amon de Karnak. (C) Marie-Paule Jung. 


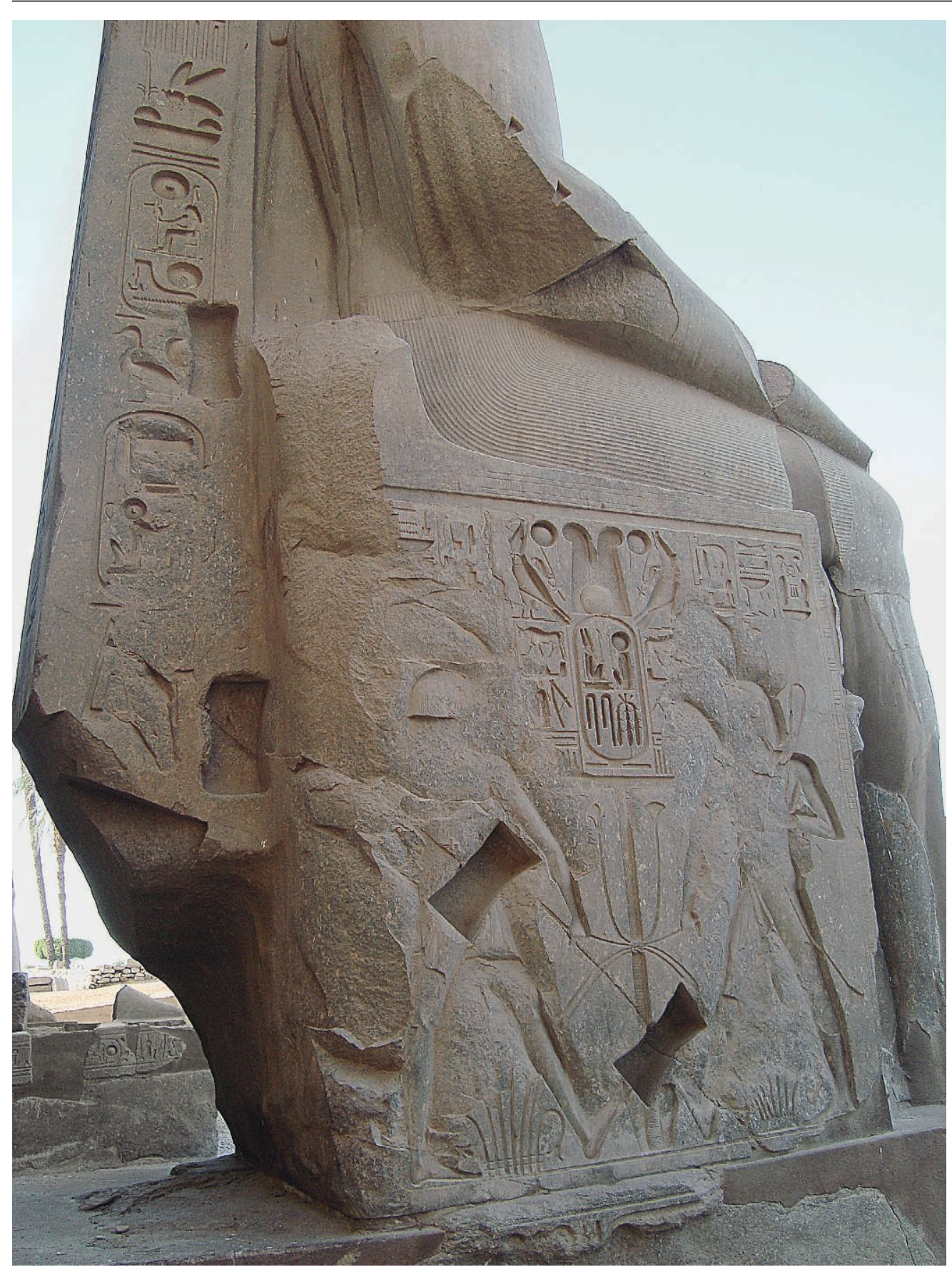

Fig. 4. Colosse de

Ramsès II situé au côté ouest de la face nord du I ${ }^{\text {er }}$ pylône du temple de Louqsor.

(c) Marie-Paule Jung.

C'est le cas d'un groupe statuaire en grauwacke représentant Thoutmosis III et Amon conservé au Musée égyptien du Caire ${ }^{21}$ (fig. 1). Plusieurs techniques différentes ont été employées lors de sa restauration. Le torse, le bras droit et les jambes de la divinité ont été retaillés directement dans l'épaisseur restante du bloc primitif, apparaissant ainsi en retrait par rapport au roi. Les dommages subis ne permettant pas de restituer la totalité du dieu avec cette méthode, des pièces supplémentaires ont été taillées puis fixées à l'ensemble. Une nouvelle tête et une couronne ont ainsi été conçues, sans doute équipées de tenons qui venaient s'insérer dans les deux mortaises trapézoïdales visibles au niveau de l'appui dorsal. Georges Legrain, inventeur de la statue, signale que la coiffe du dieu, habituellement composée de deux hautes plumes, n’a pas été retrouvée. La première publication de l'objet suggère que la tête, quant à elle, a été découverte lors des fouilles, puisque son absence sur la planche représentant le groupe y est précisée. De plus, Legrain signale la disparition de la barbe, information qui ne serait pas pertinente en l'absence d'une tête ${ }^{22}$. L'épaule et le bras gauche, ainsi que les pieds sont également des éléments nouvellement façonnés, fixés à l'aide d'adhésifs et de tenons ${ }^{23}$. La figure d'Amon ainsi restaurée prend le style caractéristique de la période postamarnienne, se manifestant notamment par un modelé très souple du torse et une ceinture de pagne qui remonte très haut sur les hanches. Cette apparence tranche d'ailleurs si 
nettement avec celle du roi qui l'accompagne qu'on est tenté d'y voir la volonté d'afficher ostensiblement l'acte de restauration. On sait par ailleurs que les Égyptiens n'avaient aucune difficulté à imiter le style des productions d'une période antérieure. De nombreuses sculptures, en particulier aux époques tardives, s'inspirent de modèles plus anciens dont elles empruntent certaines caractéristiques. La volonté d'exposer ouvertement une restauration sans chercher à la masquer trouverait un parallèle dans la pratique bien attestée de graver un texte de dédicace commémorant des restaurations sur les monuments concernés ou à leur proximité ${ }^{24}$.

La restauration du groupe de Thoutmosis III est donc datable non seulement par les critères historiques évoqués ci-dessus, mais aussi par le style de la représentation d'Amon. Si les attaques menées à l'encontre de ce dernier visaient à l'empêcher d'agir ${ }^{25}$, sa restauration permet de lui restituer pleinement sa capacité d'action. Une telle intervention était probablement suivie de rituels tels que celui de l'ouverture de la bouche, qui permettait d'activer magiquement les images égyptiennes après leur création ${ }^{26}$.

D'autres exemples de restauration de divinités à l'époque post-amarnienne sont connus: on peut ainsi citer un second groupe figurant Thoutmosis III et Amon, conservé au Musée égyptien du Caire (CG 42065), un buste d'Amon du musée du Louvre (E 10377), créé afin de compléter une représentation du dieu ou encore une sculpture d'Amenemhat I Ir exposée au sud de la «cour du Moyen Empire» du temple d'Amon de Karnak $^{27}$.

\section{Conclusion}

Les réfections effectuées par les anciens Égyptiens pouvaient répondre à la nécessité d'entretenir et de préserver les monuments, mais aussi au besoin de renouveler l'efficacité d'une représentation, selon des logiques propres à la fonction des images pharaoniques. Elles sont aussi, tout comme la création de nouveaux monuments, un moyen de témoigner sa piété envers les dieux et les ancêtres. Au-delà de ces aspects, la restauration permet de préserver des sculptures représentatives d'un passé plus ou moins éloigné. Dans la civilisation pharaonique, qui répète inlassablement les mêmes modèles issus de temps mythiques, l'ancienneté d'un objet constitue un gage de son efficacité.

Les textes égyptiens nous rappellent qu'il incombe à chacun de préserver les monuments et la mémoire de ses prédécesseurs, et celui qui s'acquitte de cette tâche en retire bénéfice. Le commanditaire d'une restauration se rattache au dieu ou à l'homme dont il rénove le monument, lequel saura sans doute le récompenser selon un système de don et de rétribution omniprésent dans la religion égyptienne. Lorsque les restaurations sont accompagnées d'inscriptions commémoratives, le lien entre le restaurateur et le bénéficiaire de l'intervention est d'ailleurs souligné.

\section{Remerciements}

Il m'est agréable de remercier ici Brigitte Bourgeois, conservateur en chef du patrimoine au Centre de Recherche et de Restauration des Musées de France, pour m'avoir proposé de participer à ce numéro de la revue Technè. Merci également à Christophe Barbotin, conservateur en chef au département des Antiquités égyptiennes du musée du Louvre, pour ses conseils et relectures.
Notes

1. Voir Assmann, 2003, p. 171.

2. Pour une définition moderne de ces termes appliqués aux biens culturels, voir Bergeon Langle et Curie, 2009, p. 1126-1127 et 1135 .

3. Voir à ce propos le groupe de Thoutmosis III et Amon présenté infra.

4. Pour un aperçu du vocabulaire égyptien, voir Jung, 2012, p. 64-69.

5 . Ce thème est l'objet des recherches que nous effectuons dans le cadre d'un troisième cycle à l'École du Louvre.

6. La question des éléments rapportés et des statues dites composites fait l'objet d'un développement dans Jung, 2012, p. 34-41.

7. Les circonstances de découverte du nez de la statue de Bakenkhonsou n'ont pas été précisées par son inventeur, G. Legrain, qui le retrouva probablement encore fixé au visage de la statue, voir Legrain, 1909, p. 21-23, pl. XVIII.
8. C'est le cas pour les statues d'Amenhotep III et de Bakenkhonsou citées supra.

9. Une statue en granite gris représentant le dieu-faucon Houroun et Ramsès II enfant, conservée au Musée égyptien du Caire (JE 64735), comporte une pièce de restauration au niveau de la face du faucon, façonnée dans un calcaire de coloris similaire à celui du granite dans lequel le groupe est taillé.

10. On peut signaler à ce propos une tête d'Aménophis III dont l'extrémité du nez, brisée, a été complétée par un mortier granuleux rougeâtre formé probablement d'un mélange de sable, d'argile et d'un liant de type résineux, voir Berman, 1999, p. 222224.

11. Voir l'exemple des criosphinx cités infra.

12. Voir le groupe d'Amon et Thoutmosis III présenté infra, dont il est possible de dater la restauration.

13. Pour un aperçu de ces textes concernant les édifices et les sculptures, voir Grallert, 2001, p. 29-30, 67-78.
14. J'ai pu observer les statues des temples de Karnak dans des conditions privilégiées au cours d'un stage de longue durée effectué en 2013 au Centre FrancoÉgyptien d'Étude des Temples de Karnak (CSA/USR 3172 du CNRS). Que ses directeurs, Christophe Thiers et Abdel Hakim Karar, ainsi que toute l'équipe du CFEETK, en soient ici remerciés.

15. Voir Cabrol, 1995, p. 4-6. L'usage de plâtre pour boucher des lacunes dans le grès est bien attesté à Karnak, en particulier sur les murs du temple où il est souvent encore visible, parfois recouvert de polychromie.

16. A. Cabrol a identifié jusqu'à sept couches de peinture successives à certains emplacements. Des criosphinx présentent des traces de couleur bleue, rouge, jaune, et de blanc et noir. Certaines statues semblent avoir été entièrement recouvertes de jaune à un moment donné, voir Cabrol, 1995, p. 10-11.

17. On sait par ailleurs que ces criosphinx ont été déplacés au cours de l'Antiquité, notamment lors de réaménagements architecturaux peut-être 
contemporains de la rénovation de ces statues, voir Cabrol, 1995, p. 2.

18. Cette sculpture a bénéficié d'une étude récente, voir Barbotin, 2012, et en particulier p. 37-38.

19. Un crampon est une pièce prenant la forme de deux queues-d'aronde liées par leur plus petit côté. Il permet de solidariser deux éléments comportant chacun une mortaise en queue-d'aronde. Des exemples en bois, en pierre et en métal sont connus.

20. Cette stèle est conservée au musée égyptien du Caire (CG 34183), pour une traduction de son inscription, voir Bennett, 1939.

21. Le groupe a été découvert à Karnak en 1904 lors des fouilles de la «Cachette» de la cour du VII ${ }^{\mathrm{e}}$ pylône du temple d'Amon, voir Legrain, 1906, p. 39, pl. XXXIX.

22. Voir Legrain, 1906, p. 39: "Parmi

il manque encore les hautes plumes du casque, la barbe et une partie du bras. La tête d'Amon ne figure pas sur la planche XXXIX. » Nous ignorons si la tête du dieu est encore conservée.

23. Ces pièces ont sans doute été de nouveaux fixées à la statue peu après sa découverte.

24. Ces inscriptions concernent le plus souvent des édifices ou des reliefs gravés sur les murs d'un temple ou sur une stèle. Pour des exemples, on se reportera à Grallert, 2001, p. 67-78.

25. La destruction partielle ou totale d'une image permet, selon les croyances des anciens Égyptiens, d'empêcher le personnage représenté d'agir de façon négative, voir Sauneron, 1966, p. 457.

26. À propos du rituel d'ouverture de la bouche, voir Assmann, 2003, p. 456-466. 27. Le groupe de Thoutmosis III a été publié dans Legrain, 1906, p. 38-39, pl. XXXVIII. Pour le buste d'Amon, voir Barbotin, 2011. Une étude de la restauration du groupe d'Amenemhat $\mathrm{I}^{\text {er }}$ est en préparation par l'auteur du présent article.

\section{Bibliographie sélective}

Assmann J., 2003, Mort et au-delà dans l'Égypte ancienne, éditions du Rocher, Paris.

Barbotin, Ch., 2011, «Un cas de restauration antique au musée du Louvre", dans Valbelle, D. et Yoyotte J.-M. (dir.), 2011, Statues démembrées et reconstituées: hommage à Charles Bonnet, Presses de l'Université de Paris Sorbonne, Paris.

Barbotin Ch., 2012, "La "tête Salt": un chefd'œuvre de la sculpture égyptienne du Moyen Empire ", Revue des musées de France 1, p. 34-45.

Bennett J., 1939, "The restoration inscription of Tutankhamun", Journal of Egyptian Archaeology 25, p. 8-15.

Bergeon Langle S. et Curie P. (dir.), 2009, Peinture et dessin. Vocabulaire typologique et technique, éditions du patrimoine, Centre des monuments nationaux, Paris.

Berman L. (dir.), 1999, The Cleveland Museum of Art: Catalogue of Egyptian Art, Hudson Hills Press, New-York, p. 222-224.

Brand P., 2010, "Reuse and Restoration", dans Wendrich W. (éd.), UCLA
Encyclopedia of Egyptology, http:// digital2.library.ucla.edu/viewItem. do?ark=21198/zz002311q4 (consulté le 17 février 2014)

Cabrol A., 1995, «Les criosphinx de Karnak: un nouveau dromos d'Amenhotep III ", Cahiers de Karnak 10, p. 1-28, pl. I-IV.

Fischer H. G., 1974, "The Mark of a Second Hand on Ancient Egyptian Antiquities", Metropolitan Museum Journal 9, p. 5-34.

Grallert S., 2001, Ägyptische Bau-und Restaurierungsinschriften von den Anfängen bis zur 30. Dynastie, Abhandlungen des Deutschen Archäologischen Institut Kairo, 18, Achet Verlag, Berlin.

Legrain G., 1909, Statues et statuettes de rois et de particuliers II. Catalogue général des antiquités égyptiennes du Musée du Caire, Imprimerie de l'Institut Français d'Archéologie Orientale, Le Caire.

Legrain G., 1906, Statues et statuettes de rois et de particuliers I. Catalogue général des antiquités égyptiennes du Musée du Caire, Imprimerie de l'Institut Français d'Archéologie Orientale, Le Caire.

Sauneron S., 1966, Le monde du sorcier dans l'Égypte pharaonique, Sources Orientales VII, Paris.

\section{Document inédit}

Jung M.-P., 2012, La restauration de la statuaire en Égypte ancienne. Mémoire de Deuxième Cycle de l'École du Louvre, non publié, Paris. 